\title{
Detection of Elemental Mercury in Abdominal Wall Soft Tissue
}

\author{
Linda S. Ellis, MDa,c, Michael E. Mullins, $M D^{b}$, Nancy Galvin, PhD', Anthony J. Scalzo, MD ${ }^{a, c, d}$
}

aSaint Louis School of Medicine, Cardinal Glennon Children's Medical Center, St. Louis, MO

bWashington University School of Medicine, St. Louis, MO

'Saint Louis University School of Medicine, St. Louis, MO

dMissouri Regional Poison Center, St. Louis, MO

\begin{abstract}
Case Report: A 19-year-old male with an unremarkable medical history presented with his father, who requested an evaluation of a pathology specimen from a reported "lump" under the skin in the middle of the son's lower abdomen. The lump had been excised by a surgeon approximately 3 months prior, per parental request. Upon gross inspection, the specimen appeared to contain small metallic droplets. The patient denied self-injection of any metals, including mercury, despite the results of a 24-hour urine heavy-metal screen (without chelation) that revealed an elevated concentration of mercury ( $87.6 \mu \mathrm{g} / \mathrm{g}$ creatinine; reference range for nonexposed adults: $<4 \mu \mathrm{g} / \mathrm{g}$ creatinine). Confirmatory analysis of the tissue sample included gross and microscopic examination, electron microscopy using secondary and backscattered electron imaging modes, and energy dispersive x-ray spectrum analysis of isolated tissue particles. Grossly, the tissue had small silver spherules suggestive of elemental mercury; these droplets were identified histologically with associated foreign body reaction. Numerous smooth, round-to-oval particles scattered randomly throughout the tissue were identified ultrastructurally, which produced an x-ray energy spectrum corresponding to mercury.

Discussion: Elemental mercury is liquid at room temperature and may be injected into the body for recreational, psychiatric, and other purposes. Isolated cases of mercury injection following accidents with broken thermometers have been reported, as well as cases of elevated metallothionein concentration following human gingival amalgam tattoos.

Conclusion: In cases of surreptitious injection, histology and ultrastructural evidence may be used to confirm the presence of mercury.
\end{abstract}

\section{INTRODUCTION}

Depending on the form, mercury compounds have varying toxic potentials [1]. Elemental (liquid) mercury and mercury vapor are poisonous and can enter the body through the respiratory tract by inhalation, the gastrointestinal tract through oral ingestion, or through the skin and soft tissues through injection or contamination through unbroken skin. Dermal contact and oral ingestion usually result in very small or negligible absorption, and rarely result in toxicity; however, rare cases of mercury poisoning secondary to ingestion and skin absorption have been reported [2]. Inhalation and injection may result in a high degree of absorption and ensuing toxicity, as mercury accumulates in the body due to a slow rate of elimination [3]. In addition to the well-described pulmonary and CNS effects, mercury toxicity has been associated with several diseases and conditions, many in a correlative, if not causal, manner. A few of the reported conditions include autoimmune disease, neurological disorders such as amyotrophic lateral

Keywords: mercury, heavy metal toxicity, foreign body, soft-tissue injury, electron microscopy, energy dispersive x-ray spectrum Notes: There was no outside funding of any kind used for this study.

The authors have no potential financial conflicts of interest to report.

Corresponding Author: Anthony J. Scalzo, MD, 1465 S. Grand Blvd., St. Louis, MO 63104-1095. Email: scalzoaj@slu.edu 
sclerosis-like motor neuron disease, autism, Parkinson disease, thrombocytopenia, dermatological disease (acrodynia), renal tubular necrosis, and glomerulonephritis [1,4-8].

Acute toxicity with most forms of mercury can be diagnosed with 24-hour urine concentration, as well as random urine specimens evaluating mercury-to-creatinine ratio. Blood concentration tends to rise rapidly after acute mercury exposure, but may not reflect tissue burden as the level also tends to fall rapidly. Blood concentrations, rather than urine concentrations, may be useful indicators of acute exposure and tissue burden to organomercurials, as these compounds are primarily excreted in the bile, rather than in the urine. Ultimately, both urine and blood mercury concentrations, whether elevated or within normal ranges, may not reflect the extent of mercury in the body, and therefore poorly reflect the degree of toxicity present $[5,8]$. Hair analysis for mercury may help identify chronic methylmercury exposure, but is infrequently used due to the potential for contamination of the hair by mercury in the ambient air, as well as the potential for uneven distribution of metal in hair $[1,8]$.

\section{CASE REPORT}

A 19-year-old male with an unremarkable medical history reported a palpable "lump" under the skin in the midline of his lower abdomen, $3 \mathrm{~cm}$ in greatest dimension. No other pertinent findings were appreciated by physical exam; no psychological testing was performed. Approximately 2 years after the appearance of this lump, it was excised at the family's request. The gross finding of metallic fragments within yellow-tan fibrofatty tissue (Figure 1) was confirmed by microscopic examination. The metallic substance was present within the deep and mid-reticular dermis with associated foreign-body reaction (Figure 2). The social history was only significant for the fact that the father was a $\mathrm{PhD}$ chemist. Due to the curious metallic fragments embedded within the soft tissue and the patient's denial that he had self-injected them, the patient's family pursued further evaluation of the shiny material.

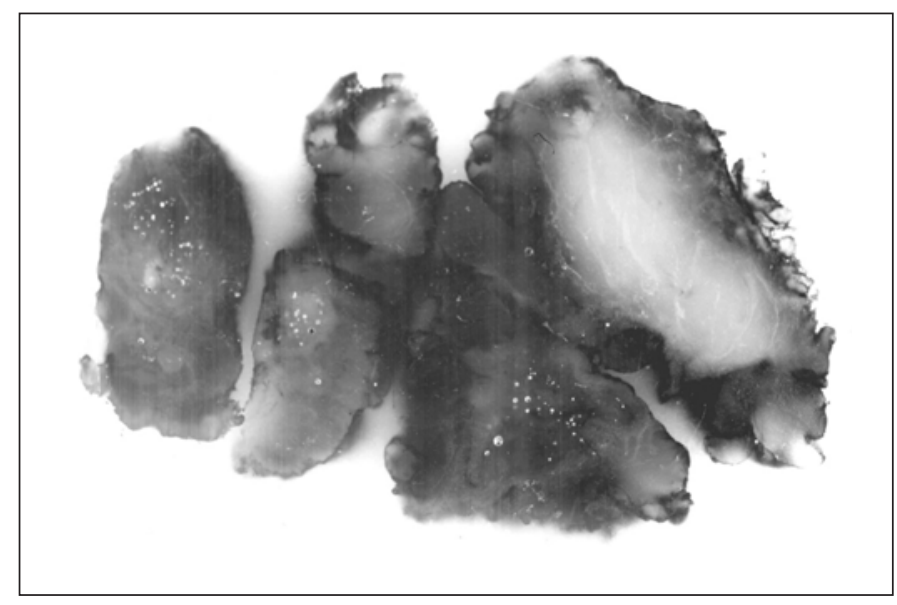

Figure 1: Paraffin-embedded soft tissue block with refractile silver droplets.

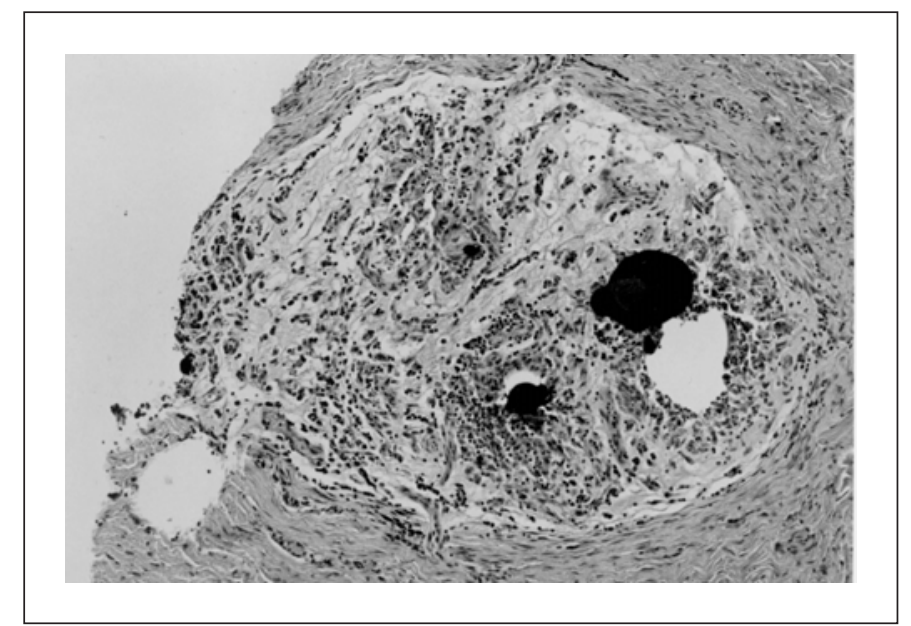

Figure 2: Droplets of foreign material with associated foreignbody-type giant cells and hemosiderin-laden macrophages (hematoxylin and eosin; original magnification: 10X).

By scanning electron microscopy, micrographs photographed in the secondary electron imaging mode (SEI) show numerous smooth, round-to-oval-shaped particles scattered randomly throughout the tissue (Figure 3). These particles ranged from 15 to $50 \mu \mathrm{m}$ in diameter. All of the round particles produced an $\mathrm{x}$-ray energy spectrum corresponding to mercury (Figure 4).

In addition to the smooth, round particles, backscattered electron imaging revealed occasional smaller, irregularly-shaped particles of heavier atomic weight than the surrounding biological tissue. X-ray spectra of these particles indicated that they were iron $(\mathrm{Fe})$, silicon $(\mathrm{Si})$, and magnesium $(\mathrm{Mg})$.

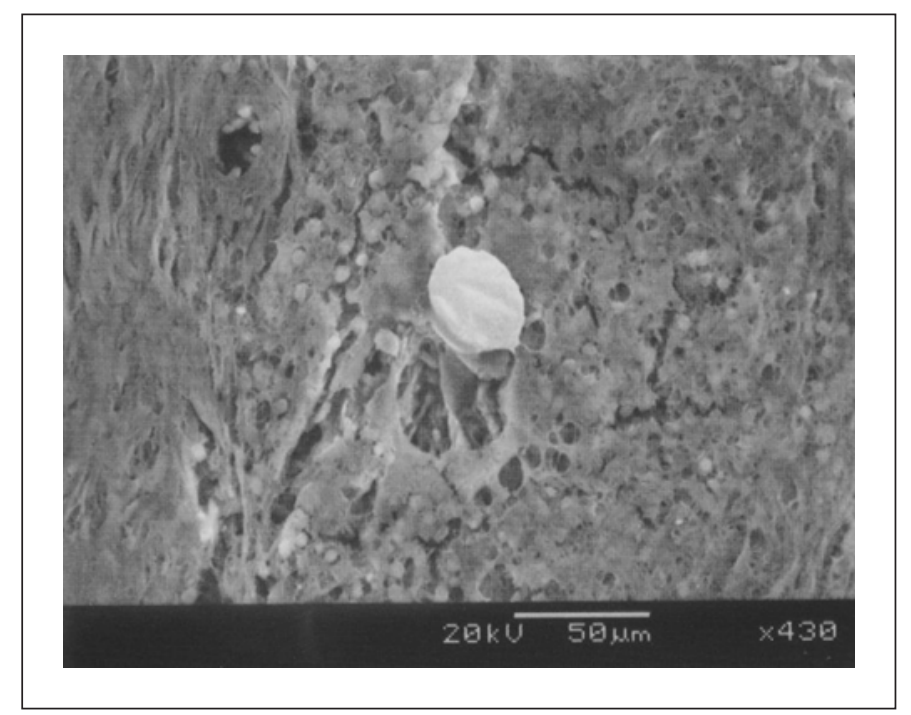

Figure 3: Ultrastructural appearance of mercury deposits with smooth, rounded surfaces (SEM; original magnification: 430,000X). 


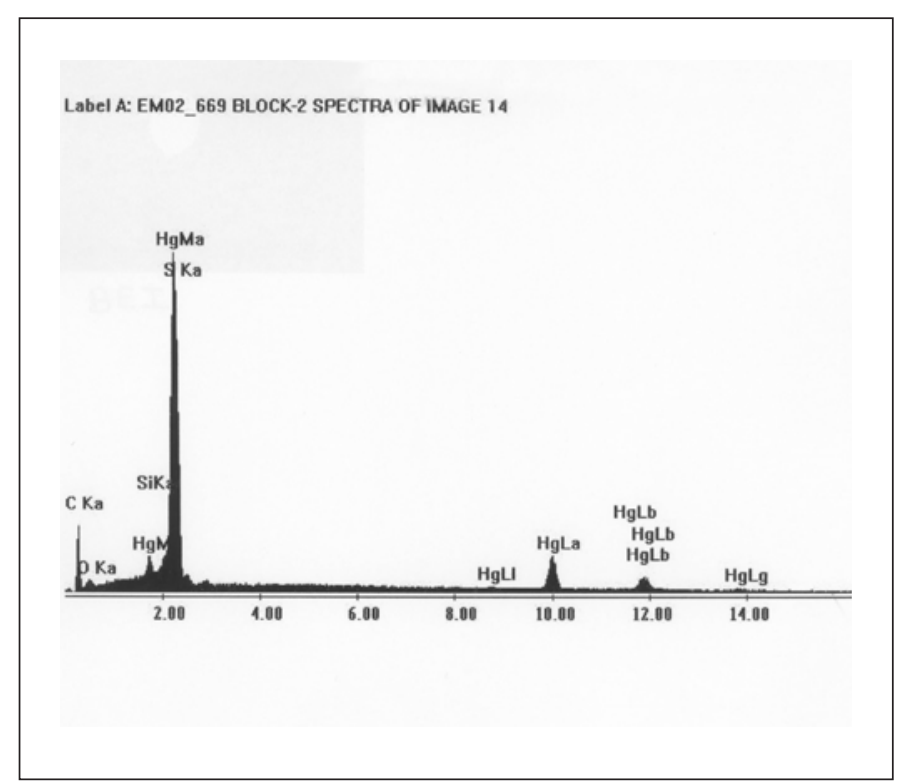

Figure 4: Energy dispersive $x$-ray spectrum of soft-tissue droplets, indicating the presence of mercury.

Approximately 10 weeks postoperatively, the patient's random urine heavy metal screen revealed elevated mercury $(87.6 \mathrm{~g} / \mathrm{g}$ creatinine; reference range for nonexposed adults: $<4 \mathrm{~g} / \mathrm{g}$ creatinine). Blood mercury concentration was undetectable. His serum creatinine and a complete blood count were entirely within normal limits. One month later, the random urine mercury-to-creatinine ratio was still elevated $(11.9 \mathrm{~g} / \mathrm{g})$. Due to the persistence of elevated mercury concentration and the potential for mercury toxicity, as well as at the father's insistence, the patient was begun on chelation therapy with meso-2,3-dimercaptosuccinic acid (DMSA). As expected, 2 days after initiation of therapy, a random urine mercury-to-creatinine ratio was increased $(61.7 \mathrm{~g} / \mathrm{g})$, consistent with tissue mercury mobilization and increased mercury excretion. The following month, 11 days after completion of chelation therapy, the random urine mercury-to-creatinine ratio remained elevated, but was decreased from previous results $(20.4 \mathrm{~g} / \mathrm{g})$. The patient continues with regular follow-up with a toxicologist and his primary physician and remains healthy without demonstrable sequelae of mercury toxicity.

\section{DISCUSSION}

Mercury is well known to be highly volatile, with toxicity primarily dependent on the method of exposure. Inhalation of elemental mercury, via industrial exposure, smelting, or improper disposal of liquid mercury may result in up to $80 \%$ absorption with pulmonary, central nervous system, renal, and hepatic toxicity, and a high rate of associated morbidity and occasional mortality. Elemental mercury accumulates in these systems due to its lipid-soluble nature. Mercurials react with sulfhydryl radicals and bind to proteins, including hemoglobin and enzymes, affecting several important systems including cytochrome C oxidase syn- thesis and oxidative mitochondrial phosphorylation $[3,7]$. Depending on its form, mercury's overall half-life ranges from 2 to 70 days [5]. Indeed, several reports of elevated levels of mercury in urine and blood have been documented days, weeks, months, and even years after a single exposure has taken place $[6,9]$. While the lethality of mercury vapor is well documented, several reports of death associated with mercury injection have been published [9]. In most instances, the deaths are related either to direct intravenous (IV) injection or primary soft-tissue injection with subsequent infiltration of the lymphatic and/or vascular channels. The injections may be the result of suicide or homicide attempts, accidental injection from broken thermometers, or premeditated injection into soft tissues for enhancement of physical prowess or cultural rituals, such as espiritismo, a ritual originating in the Caribbean thought to prevent evil and bring good luck $[10,11]$.

Documentation of mercury exposure is obtained most reliably via 24-hour urine collection, when possible. Random urine examination for mercury itself is not often helpful except in identifying acute exposure; however, assessment of mercury-to-creatinine ratios in the urine allows for a one-time collection of specimens in an outpatient or workplace setting [12]. These ratios correlate well with total daily excretion rates as measured in 24-hour collection specimens. Due to the redistribution of mercury in the soft tissues, however, levels of urine or blood mercury do not correlate with severity of clinical toxicity $[3,7]$.

Our patient had noticed his soft-tissue abdominal mass for a "few years" prior to its surgical excision when he was 19 years old. At the time of surgical excision, no additional laboratory or radiographic studies were performed to evaluate for mercury injection and/or toxicity. His lesion was excised at a local hospital; the patient was subsequently seen in consultation at our hospital nearly 3 months later. This patient's family sought consultation with a toxicologist based on the gross appearance of the excised specimen-the silvery substance, in particular. All studies performed on our patient were done at the request of the family in order to identify the material present in the soft-tissue mass.

Histological examinations of soft-tissue mercury injection sites have been described similarly in the literature. Most cases, as ours, consist of a foreign-body-type reaction with numerous histiocytes and fibroblasts, with varying numbers of multinucleated giant cells and lymphocytes. In some studies, the lesions were associated with necrosis and abscess formation with numerous neutrophils and eosinophils. In nearly all cases, brown or black dense metallic material in globules was identified in tissue sections. One study identified spaces within the reactive tissue where the globules had been lost in tissue processing [3,8,14-17].

Only 2 studies in the literature of the past 75 years have evaluated the electron microscopic features and energy spectrum analysis of mercury. One was a retrospective study of oral amalgam tattoos in which the composition of the sample dental amalgams analyzed was largely mercury, silver, and tin, which correlated with the elements identified in the oral tattoo tissue samples [18]. In addition, iron and selenium were present in the tissue samples, but not in the dental amalgams themselves. Along with mercury, iron 
and silicon were identified in our patient's sample; the former likely a reflection of the noted hemosiderin-laden macrophages in the foreign-body reaction. The presence of silicon in our sample is of unknown etiology, but likely a contaminant associated with the method of injection and/or storage of the elemental mercury prior to injection.

Current treatment of inorganic and elemental mercury exposure and toxicity includes the use of DMSA, a water-soluble sulfhydryl-containing oral chelation agent with low toxicity. DMSA is superior to previously-used agents including N-acetylD,L-penicillamine in terms of efficacy, inhibiting the uptake of methylmercury by human red blood cells and hepatocytes, mobilizing tissue stores of mercury, and increasing its urinary excretion. In Europe and the Middle East, 2,3-dimercaptopropane sulfonate (DMPS) has been used extensively in conjunction with N-acetylcysteine and hemodialysis in a treatment regimen for multiple forms of mercury exposure $[2,7,9,10,15]$. All cases of mercury granulomas secondary to IV or subcutaneous injection require surgical excision to remove the source of mercury. These patients may or may not benefit from oral chelation $[1,13,14,16,17]$.

Toxicological examination of this patient included laboratory studies, physical and neurological exams, and further workup of the surgical pathology specimen. The patient's urinary excretion of mercury was elevated prior to chelation therapy, which itself causes mercury concentrations to rise in cases of prior exposure. As tissue redistribution of mercury precludes correlation of urine and blood levels with clinical toxicity, the patient agreed to undergo chelation therapy with DMSA as a precautionary measure. As expected, immediately following chelation, urinary mercury concentration was increased, but thereafter declined. At present, the patient remains asymptomatic and urinary mercury concentrations continue to decline. Although the laboratory and histological interpretation of the metallic soft-tissue substance has been definitively identified as mercury, the patient refuted the possibility of either accidental or purposeful injection of, or exposure to, mercury. Its presence in this young man seems to be an enigma. Psychological counseling was suggested by the medical team.

\section{REFERENCES}

1. Cole JK, Holbrook JL. Focal mercury toxicity: A case report. J Hand Surg. 1994;19A:602-603.

2. McLauchlan GA. Acute mercury poisoning. Anaesthesia. 1991;46:110-112.
3. Campbell BG. Broadsheet number 48: Mercury, cadmium and arsenic: Toxicology and laboratory investigation. Pathology. 1999;31:17-22.

4. Adams CR, Ziegler DK, Lin JT. Mercury intoxication simulating amyotrophic lateral sclerosis. JAMA. 1983;250: 642-643.

5. Clarkson TW. The toxicology of mercury. Crit Rev Clin Lab Sci. 1997;34:369-403.

6. Dell'Omo M, Muzi G, Bernard A, et al. Long-term pulmonary and systemic toxicity of intravenous mercury injection. Arch Toxicol. 1997;72:59-62.

7. Graeme KA, Pollack CV. Heavy metal toxicity, part I: Arsenic and mercury. J Emerg Med. 1998;16:45-56.

8. Kedziora A, Duflou J. Attempted suicide by intravenous injection of mercury: A rare cause of cardiac granulomas. Am J Forensic Med Pathol. 1995;16:172-176.

9. Miller AL. Dimercaptosuccinic acid (DMSA), a non-toxic, water-soluble treatment for heavy metal toxicity. Altern Med Rev. 1998;3:199-207.

10. Baum CR. Treatment of mercury intoxication. Curr Opin Pediatr. 1999;11:265-268.

11. Smith SR, Jaffe DM, Skinner MA. Case report of metallic mercury injury. Pediatr Emerg Care. 1997;13(2):114-116.

12. Roels HA, Hoet P, Lison D. Usefulness of biomarkers of exposure to inorganic mercury, lead, or cadmium in controlling occupational and environmental risks of nephrotoxicity. Ren Fail. 1999;21(3-4):251-262.

13. Bradberry SM, Feldman MA, Braithwaite RA, et al. Elemental mercury-induced skin granuloma: A case report and review of the literature. J Toxicol/Clin Toxicol. 1996;34: 209-216.

14. Netscher DT. Subcutaneous mercury granulomas (letter). J Hand Surg. 1995;20A:516-517.

15. Rachman R. Soft-tissue injury by mercury from a broken thermometer: A case report and review of the literature.

Am J Clin Pathol. 1974;61:296-300.

16. Theodorou S, Vlachos P, Vamvasakis E. Knee joint injury by mercury from a broken thermometer: Case report and review of the literature. Clin Orthop. 1981;160:159-162.

17. Yotsuyanagi T, Yokoi K, Sawada Y. Facial injury by mercury from a broken thermometer. J Trauma. 1996;40: 847-849.

18. Harrison JD, Rowley PSA, Peters PD. Amalgam tatoos: Light and electron microscopy and electron-probe microanalysis. J Path. 1977;121:83-92. 